\title{
De la invisibilidad a los saberes compartidos, una mirada desde la experiencia de lesbianas y mujeres bisexuales en México
}

CORONA, Claudia Ivette Navarro'

\begin{abstract}
o
La educación sexual en el ámbito educativo se encuentra atravesada por un sistema de género-heteronormativo, que ha contribuido a una normalización de la sexualidad, los cuerpos y los deseos. El presente artículo tiene como objetivo explorar los discursos y prácticas heteronormativas que atravesaron la experiencia educativa de lesbianas y mujeres bisexuales, y comprender cómo a partir de esto se desprendieron diversas formas de resistencias. Se trabajó a partir de entrevistas a profundidad con 7 lesbianas y 4 mujeres bisexuales que residían en la Ciudad de México y el Estado de México. Como resultados se encontró una construcción de dispositivos que sostienen la heterosexualidad y dicotomía de género, dando lugar a doble proceso de invisibilización, así como a un conjunto de resistencias individuales y colectivas.
\end{abstract}

Orientación sexual. Educación sexual. Ciudadanía sexual

\section{Resumo}

A educação sexual no campo educacional é atravessada por um sistema heteronormativo de gênero, que contribuiu para uma normalização da sexualidade, dos corpos e dos desejos. O objetivo deste artigo é explorar os discursos e práticas heteronormativas que perpassam a experiência educacional de lésbicas e mulheres bissexuais, e entender como diferentes formas de resistência emergiram disso. Trabalhamos com entrevistas em profundidade com 7 lésbicas e 4 mulheres bissexuais residentes na Cidade do México e no Estado do México. Como resultado, foi encontrada uma construção de dispositivos que apóiam a heterossexualidade e a dicotomia de gênero, dando origem a um duplo processo de invisibilidade, bem como a um conjunto de resistências individuais e coletivas.

\section{Orientação sexual. Educação sexual. Cidadania sexual}

\section{Introducción}

A la sexualidad se le ha otorgado una importancia central en sociedades modernas, llegando a construir identidades y modos de vida a partir de las prácticas y experiencias sexuales (FOUCAULT, 2011; WEEKS, 1998), los cuales han sido jerarquizados, al respecto Gayle Rubin en su texto Reflexionando sobre el sexo: notas para una teoría radical de la sexualidad (1989), propone que existe una

1 Dra. en Psicología. Profesora en la Facultad de Psicología, UNAM. Especialista en feminismo, diversidad sexual y derechos humanos, claudianavarrocorona@gmail.com 
jerarquización de la sexualidad en buena y mala, ambas perfectamente trazadas; en la cima de la jerarquía se encuentra el sexo bueno, el cual corresponde a las personas heterosexuales casadas que tienen una sexualidad monógama y procreadora en casa. Debajo de la línea, a las parejas heterosexuales no casadas, sucesivamente a los heterosexuales promiscuos, a quienes se masturban, y en el límite de la respetabilidad a las parejas estables de gays y lesbianas, a las lesbianas en el bar y por debajo, los gays promiscuos en saunas y parques.

En lo peor, donde tiene lugar el sexo malo se ubica a los travestidos, los transexuales, los fetichistas, los sadomasoquistas, a quienes tienen sexo por dinero y quienes optan por el sexo intergeneracional. En este lugar la sexualidad es asociada con lo anormal, lo antinatural, lo dañino, lo pecaminoso, lo extravagante, y por ende se coloca por debajo de la respetabilidad y el reconocimiento.

Es así que en la configuración de ambos espacios es posible ubicar cualquier práctica o experiencia sexual, mientras que en el espacio del sexo bueno encontramos a todas aquellas personas que gozan de respetabilidad, reconocimiento e inclusive de ciudadanía, en el lugar del sexo malo encontramos a todas aquellas personas abyectas, inteligibles, que viven de manera permanente en la censura e incluso sin derechos.

Diversas autoras y autores (AMUCHÁSTEGUI y RIVAS, 2004; CAMPERO, KENDALL, CABALLERO, MENA y HERRERA, 2010; CARÍAS, 2006; GÜIDA, RAMOS y VITALE, 2006; LAGARDE, 2011; ORTIZ-ORTEGA, RIVAS-ZIVY, HUERTA, SALAZAR y GÓMEZ-GONZÁLEZ, 2004; RUBIN, 1989; SÁNCHEZ, 2009) han coincidido en señalar que en esta construcción social de la sexualidad las mujeres son vistas en relación a la reproducción y maternidad que a la vez amparan su reputación y validez, pues hay otras que al ser leídas como otro tipo de mujer, se les otorgan cualidades de seducción y erotismo, que aparentemente las coloca como sujetos de sexualidad, pero que ciertamente son vividas como objetos de placer para los otros -en específico los varones-. En este esquema se ha establecido que no existe una sexualidad sin penetración. De ahí que no se reconozca la masturbación, ni el sexo entre mujeres como actividades propiamente sexuales (CASTAÑEDA, 2002).

La sexualidad en la educación mexicana comenzó a tener cabida en 1974, momento en el que se caracterizó por tener un enfoque vinculado a la reproducción. De acuerdo a Rodríguez (2004) se componía de dos momentos, el primero en quinto año de primaria a partir de impartir temas como el desarrollo sexual y la reproducción humana, y el último en la secundaria al hablar sobre la prevención de embarazos e Infecciones de Transmisión Sexual (ITS). En el mismo año, tuvo lugar la Conferencia Internacional sobre la Población y el Desarrollo en Bucarest, en donde se reconoció el papel del Estado como garante de los derechos reproductivos, incluyendo el acceso a la información y métodos de control natal 
(SÁNCHEZ, 2002). Es posible ubicar este momento en la primera etapa de la discusión y desarrollo de los derechos sexuales y reproductivos, el movimiento por la planificación familiar, durante el cual la lucha social se centró en superar las barreras legales, culturales y religiosas que impedían a las personas recurrir a medios para controlar su fecundidad (FIGUEROA, 1995).

Un segundo y tercer momento, de acuerdo a Rodríguez (2004) es posible ubicarlos en 1982 y 1994, los cuales, marcan un alejamiento de la visión reproductiva. En 1982, la educación sexual se vio impactada por la lucha contra el vih/sida, produciendo un espacial énfasis en el uso del condón. Mientras que en 1994, el enfoque estuvo enfocado al género y, los derechos sexuales y reproductivos. Ambos momentos es posible ubicarlos dentro de la discusión de los derechos sexuales y reproductivos en dos movimientos en primer lugar por la salud reproductiva el cual, no sólo se basa en el control de la fecundidad, además considera la habilidad de la persona y de la población para reproducirse, para regular su fecundidad así como para practicar y disfrutar sus relaciones sexuales. $Y$ por último, en el movimiento por el empoderamiento de la mujer y justicia social; desde esta perspectiva el proceso de adquisición de poder por parte de las mujeres incluye la autodeterminación reproductiva como parte y como un medio, más que como un fin en sí misma (FIGUEROA, 1995).

Con ello innegable el avance logrado en cuanto a la educación sexual y la legitimación y consolidación de los derechos sexuales y reproductivos, sin embargo, es necesario hacer un análisis crítico que aporte nuevas rutas. En este sentido, en primer lugar es necesario hacer una distinción clara entre los derechos sexuales y reproductivos. Cervantes-Carson y Citeroni (2008) mencionan que los derechos sexuales han sido subordinados a los derechos reproductivos, reduciendo con ello la sexualidad a la reproducción. Desde los derechos sexuales se rompe la relación directa entre sexualidad y reproducción, dando espacio a la vivencia del erotismo, el placer y el deseo en la experiencia sexual, enfatizándose un proceso de autonomía en el ejercicio de la sexualidad.

Otro de los ejes críticos en la construcción de los derechos sexuales es la invisibilidad de las personas no heterosexuales en la mayoría de las declaraciones. Al respecto Girard (2009) menciona que fue en la Conferencia de Salud Reproductiva y Justicia, en Río de Janeiro (1994), donde por primera vez los derechos de las lesbianas fueron discutidos explícitamente, sin embargo, en Beijing (1995) no fue posible incluir en el párrafo 227 (que trata de las múltiples formas de discriminación contra las mujeres) una mención explícita a la orientación sexual (CORRÊA, 2008).

Tomando en cuenta los avances y rutas críticas para hablar de la educación sexual y los derechos sexuales y reproductivos en México, el presente artículo tiene 
como objetivo explorar los discursos y prácticas heteronormativas que atravesaron la experiencia educativa sobre la sexualidad en lesbianas y mujeres bisexuales, y comprender cómo a partir de esto se desprendieron diversas formas de resistencias.

\section{Método}

\subsection{Participantes}

En este estudio colaboraron de forma voluntaria y con consentimiento informado 11 mujeres que en el momento de la entrevista residían en la Ciudad de México y el Estado de México. El criterio de participación fue que tuviesen la mayoría de edad cumplida (en México se establece de los 18 años en adelante). Para tener acceso a este grupo de mujeres, y considerando la dificultad en torno a hablar de las temáticas abordadas, el procedimiento para congregar a las participantes implicó ir a organizaciones civiles feministas y de defensa de los derechos humanos de personas LGBTTTI, en donde había alguna persona conocida que podía extender la invitación hacia el resto de mujeres.

\subsection{Procedimiento}

En términos metodológicos se partió de una postura feminista, específicamente posicionándome desde una mirada analítica interseccional y queer; la primera en cuanto que el análisis realizado toma en consideración que las identidades y las experiencias de las personas son complejas y enmarañadas en función de las múltiples categorías que intersectan las experiencias de vida (Platero, 2012), y queer porque teóricamente parto de una mirada crítica que reconoce la necesidad de romper la lógica binaria, dualista y heteronormada que ha predominado en la comprensión de las sexualidades y los cuerpos; así, desde esta mirada pretendo conocer mejor las sexualidades que social y culturalmente son consideradas "abyectas", pero a su vez dar cuenta de que todas las personas están atravesadas por complejas relaciones de poder y privilegio (PLATERO, 2014). E estudio fue de corte cualitativo y comprensivo, y la recolección de datos se hizo a través de entrevistas semiestructuradas a profundidad (KVALE, 2008). En términos del análisis, para este artículo se retomó el tema de educación sobre la sexualidad e indague las experiencias de opresión y resistencia, partiendo desde el feminismo, la interseccionalidad y la teoría queer. En cuanto a las consideraciones éticas, fue indispensable el uso del consentimiento informado, el anonimato, la confidencialidad, la canalización de las participantes que lo solicitaron, así como la reflexión sobre mi implicación en la investigación, en el cruce como investigadora y mujer con una identidad erótica-afectiva diversa. 


\section{Resultados}

Las 11 participantes son mujeres $^{2}$ totalmente diferentes, muchas de ellas profesionistas o estudiantes, con acceso a un nivel educativo de licenciatura. Dos de las participantes no tenían empleo en el momento del estudio. De las participantes, aquellas que se ubican como solteras y en relación de noviazgo viven con sus familias.

Tabla1. Características generales de las entrevistadas

\begin{tabular}{|c|c|c|c|c|c|}
\hline Seudónimo & Edad & Localidad & Escolaridad & $\begin{array}{c}\text { Situación } \\
\text { Civil }\end{array}$ & $\begin{array}{c}\text { Identidad erótico- } \\
\text { amorosa }\end{array}$ \\
\hline Viviana & 27 & $\begin{array}{l}\text { Ciudad de } \\
\text { México }\end{array}$ & $\begin{array}{c}\text { Estudiante } \\
\text { universitaria }\end{array}$ & Noviazgo & Lesbiana \\
\hline Lucía & 26 & $\begin{array}{l}\text { Estado de } \\
\text { México }\end{array}$ & Licenciatura & Soltera & Lesbiana \\
\hline Verónica & 27 & $\begin{array}{l}\text { Ciudad de } \\
\text { México }\end{array}$ & Licenciatura & Noviazgo & Lesbiana \\
\hline Miriam & 30 & $\begin{array}{l}\text { Estado de } \\
\text { México }\end{array}$ & Licenciatura & Soltera & Lesbiana \\
\hline Alicia & 31 & $\begin{array}{l}\text { Ciudad de } \\
\text { México }\end{array}$ & Licenciatura & $\begin{array}{l}\text { Vive en } \\
\text { pareja }\end{array}$ & Lesbiana \\
\hline Fátima & 40 & $\begin{array}{l}\text { Ciudad de } \\
\text { México }\end{array}$ & Licenciatura & $\begin{array}{l}\text { Vive en } \\
\text { pareja }\end{array}$ & Lesbiana \\
\hline Mariana & 41 & $\begin{array}{l}\text { Ciudad de } \\
\text { México }\end{array}$ & Preparatoria & Noviazgo & Lesbiana \\
\hline Tamara & 20 & $\begin{array}{l}\text { Ciudad de } \\
\text { México }\end{array}$ & $\begin{array}{l}\text { Estudiante } \\
\text { Universitaria }\end{array}$ & Noviazgo & Bisexual \\
\hline Alejandra & 31 & $\begin{array}{l}\text { Ciudad de } \\
\text { México }\end{array}$ & Licenciatura & Noviazgo & Bisexual \\
\hline Lidia & 36 & $\begin{array}{l}\text { Ciudad de } \\
\text { México }\end{array}$ & Licenciatura & $\begin{array}{l}\text { Vive en } \\
\text { pareja }\end{array}$ & Bisexual \\
\hline Samantha & 58 & $\begin{array}{l}\text { Ciudad de } \\
\text { México }\end{array}$ & Licenciatura & $\begin{array}{l}\text { Vive en } \\
\text { pareja }\end{array}$ & Bisexual \\
\hline
\end{tabular}

Al analizar las experiencias de las participantes fue posible develar algunos de los elementos que refuerzan la desigualdad y la opresión de las mujeres lesbianas y bisexuales en el ámbito de la educación de la sexualidad en las escuelas. Sin embargo, también fue posible identificar resistencias que les han permitido subvertir el sistema y compartir experiencias de educativas fuera de los ámbitos institucionales. Si desglosamos algunos de los elementos con los cuales se

2 En las narraciones, algunas mencionaron no sentirse completamente identificadas al nombrarse como mujer, al considerar que no se apegan a los roles establecidos culturalmente, viviéndose alejadas de esta construcción, o bien, en un tránsito. Tomando en cuenta la posibilidad de utilizar mujeres como categoría política, se retoma, en plural, para visibilizar la diversidad entre las mismas. 
puede dar cuenta de cómo permean el patriarcado y la heterosexualidad obligatoria, podemos encontrar los siguientes ejes temáticos:

a) Educación sexual desde la reproducción: las entrevistadas reportaron haber recibido en dos momentos de su educación escolar información sobre sexualidad, el primero durante la educación primaria, y el último en la secundaria. La preparatoria y la universidad fueron momentos en donde relataron la inexistencia de materias que abrieran el tema.

Si bien, pareciera ser que en la primaria y secundaria eran momentos donde podrían recibir información, dado que estaba en los programas de estudio como lo mencionaba Rodríguez (2004), algunas de las entrevistadas mencionaron una omisión clara por parte de los docentes asignados a hablar sobre dicho tema, al respecto Alejandra señala:

En un año me tocó un profesor que era muy cerrado y entonces se saltó todo, todo, esa parte de educación sexual. Al siguiente año, que ya fue en tercer año, me tocó una profesora muy diferente que nos dijo de pe a pa, como sabía que no nos habían dicho nada el año pasado, fue sensible, incluso de explicarnos como se usa un condón, los métodos anticonceptivos, cual eran como las diferencias, enfermedades de manera general (Alejandra, 31 años, bisexual, licenciatura, Distrito Federal)

Lo cual hace evidente la dificultad vivida por parte de las y los docentes para hablar de la sexualidad. Como también es posible leer en el testimonio anterior cuando se mencionaba el tema, en el caso de la escuela secundaria se hacía en relación a temas como el uso correcto del condón, el uso de métodos anticonceptivos y la prevención de infecciones de transmisión sexual. Mientras que en la educación primaria aludieron a temas como el desarrollo sexual y en específico a la menstruación.

Ninguna de las participantes recuerda haber obtenido información sobre la orientación sexual, la identidad de género o la diversidad sexual, al respecto Miriam menciona:

mucho menos, no, hablar de preferencias u orientaciones sexuales diferentes a la heterosexual, no, no, era algo, no era un tema que se tocará en la secundaria ni en la preparatoria. (Miriam, 30 años, lesbiana, licenciatura, Estado de México) 
Ante este panorama limitante de acuerdo a las entrevistadas, comenzaron realizando una búsqueda en otros medios de información como libros, programas de radio, pornografía e internet, está última opción solo fue mencionada por las entrevistadas entre los 20 y 27 años. En el caso de los libros mencionaron que eran completamente informativos, llegando incluso a ser una información limitada en tanto tenía una visión reproductiva y en ocasiones religiosa. En cuanto a la pornografía, las entrevistadas relataron haberla visto en compañía con sus primos y tíos, con un contenido heterosexual, que en su momento les brindo información sobre la sexualidad de las mujeres. En cuanto al radio y principalmente el internet además de brindarles información, les permitió saber de la existencia de más lesbianas y mujeres bisexuales, así como el contexto de discriminación en que el que vive la población LGBT. Al respecto Tamara menciona:

... era reconfortante saber que habían más personas que vivían algo similar a lo que estaba viviendo, pero también aumento la preocupación, hay muchas noticias de personas que sufren, no sé cómo decirlo, sí sufren violencia por motivo de eso, por su orientación sexual. (Tamara, 20 años, estudiante universitaria, bisexual, Distrito Federal)

Cabe destacar la preocupación que menciona Tamara ya que al conocer los casos de violencia por orientación sexual, ha decido ocultar en diversos espacios su bisexualidad. Pareciera ser que ante la amenaza de una posible sanción por parte del sistema patriarcal y heteronormativo, Tamara implementa como estrategia el ocultamiento de sus deseos.

b) Heterosexualidad obligatoria en el ámbito escolar: ante este panorama se hace evidente la invisibilidad y silenciamiento de las experiencias que se salen del marco de la heterosexualidad, en donde mujeres lesbianas y bisexuales así como otras corporalidades fuera de la dicotomía de género son violentadas. Al respecto algunas de las entrevistadas relatan que la primera violencia experimentada en el ámbito escolar es que se les asuma como personas heterosexuales, ante lo cual la experimentación de la sexualidad y el género se convierte en algo difícil al realizar y nombrar.

... pienso que algunas personas dan por hecho al conocerte que eres heterosexual, entonces siempre me pregunto ¿qué pasaría si supieran que no soy?, cambiaria algo en su manera de tratarme, si son varias preocupaciones. (Tamara, 20 años, estudiante universitaria, bisexual, Distrito Federal) 
Aunado a ello existe una advertencia para no salirse del esquema heterosexual, y con ello, una normalización de los deseos y cuerpos. Está advertencia conlleva al igual que en el caso de Tamara al ocultamiento como estrategia para no vivir discriminación escolar, y con ello ha una autocensura. Al respecto Fátima menciona la siguiente experiencia:

Una amiga me dijo ten cuidado, dicen que esa chava es ¿cómo me dijo? Marimacha, algo así, como rara, quiso decir lesbiana pero yo le dije ¿a poco sí? Lo que hice fue alejarme de la chava porque me di cuenta que si sabían que yo también era así pues me iban a dejar de hablar, no la empecé a pelar, la chava se cansó y se fue, pero seguramente sí yo hubiera empezado a salir más con ella se hubiera dado algo, porque además me gustaba la chica pues, nunca más la volví a ver una vez que salí de la Prepa. (Fátima, 40 años, licenciatura, lesbiana, Distrito Federal)

Como es posible observar en el ámbito escolar son diversas las experiencias de discriminación que vivieron una vez que las entrevistadas salieron del closet, que va desde la reglamentación del cuerpo, el aislamiento, el acoso sexual hasta la salida obligada del closet. Fátima al respecto relata una experiencia, donde incluso es negado por parte de quienes ejercieron dichas conductas, que la discriminación por orientación sexual fuera la razón.

Cuando ya fue más formal (la relación que tenía con una mujer) se lo dije a mis amigos y este ellos se sacaron mucho de onda, se hizo el chisme en la escuela, hubo un distanciamiento con mis amigos por esa onda, ellos dijeron que no era por eso, pero a raíz que yo les digo que soy gay me empiezan a sacar de los equipos. (Fátima, 40 años, licenciatura, lesbiana, Distrito Federal)

Cabe destacar estos actos de discriminación también surgen por parte del personal docente. En el caso de Tamara menciona que uno de sus docentes universitarios reforzó los prejuicios hacia personas bisexuales durante la clase, ante las risas de las y los demás estudiantes en dicho momento prefirió quedar en silencio:

También este semestre un profesor hizo un comentario que lo que estaba en boga en la actualidad era ser original, y que las personas que se denominan bisexuales lo hacen para no pertenecer a una cosa, ni a otra, sino para ser originales" (Tamara, 20 años, estudiante universitaria, bisexual, Distrito Federal) 
Ante este panorama que muestra discriminación tanto por parte del personal docente, así como del alumnado, se hace evidente que el contexto escolar no es uno de los más sensibles ante el tema.

c) Actos de resistencias: Ante la falta de apertura y la existencia de actos de violencia y discriminación las entrevistadas relataron la búsqueda de espacios en donde encontraron a otras mujeres que atravesaron experiencias similares. En estos espacios tuvieron la oportunidad de conocer otras formas de vivir que les han permitido cuestionarse y deconstruirse como lo menciona Viviana:

empiezo a formar alianzas, principalmente con mujeres que son más políticas, que manejan un discurso como más de lucha que a veces no se preguntan pero que saben perfectamente cómo se están viviendo y como quieren vivir su sexualidad, sin tanta represión, sin tanto control, sin tanto sentimiento de culpa, y entonces eso me hace decir, güey no mames, ahí otro mundo, puedes acceder a el, y este compañerismo empieza a ayudarme a deconstruirme como tal, de repente me toca comenzar a viajar por otros lados, conocer a gente que viene no de mi espacio, sino de espacios súper diferentes y entonces ahí me doy cuenta que el trabajo es desde ti y poco a poco vas construyendo nuevamente ese espacio de sexualidad, que te encuentras un chingo de alianzas y que de cada una aprendes, puta, eso también, esas historias de vida me han servido bastante para ir reincorporando, reintegrando cosas y descubriendo que pedo conmigo misma." (Viviana, 27 años, lesbiana, licenciatura, Distrito Federal)

Como es posible leer, entre los temas que se abrieron a nuevas experiencias estuvo la sexualidad, convirtiéndose incluso en un espacio para tejer alianzas con otras mujeres. Estos espacios y nuevas alianzas les permitieron obtener herramientas para afrontar la discriminación y aunado a ello, tener información para compartir con otras personas LGBT. A partir de lo cual, comenzaron a generar espacios educativos desde y para las personas LGBT.

"ue una motivación desde mi propia experiencia, es necesidad de buscar, de buscar formas, en ese momento lo pensaba, para que otras personas no tuvieran que pasar por un proceso tan largo que era la percepción que tenía del mío, con tantos obstáculos y discriminación, me corrieron de mi trabajo por ser lesbiana, rechazo, un montón de cosas, que generaron heridas emocionales, que dije no quiero que ninguna persona más en el mundo, ninguna lesbiana, ninguna persona homosexual lo viva como lo tuve que vivir, 
entonces desde ahí empezó como la motivación. (Miriam, 30 años, lesbiana, licenciatura, Estado de México).

En el ámbito de la sexualidad, en el reconocimiento de la escasa información sobre las mujeres lesbianas y bisexuales, tres de las once participantes mencionaron haberse involucrado en generación de insumos así como de pláticas y talleres para hablar con otras mujeres sobre el tema, al respecto Fátima menciona:

en la organización desde hace mucho... la médica del grupo, decía no hay nada para protegernos, tampoco información de que enfermedad nos podemos contagiar, hasta la clamidia, o la cándida, estábamos hasta esas dos y era muy recurrente que alguien dijera es que tengo candidiasis, y ellas hicieron un manualito e hicimos condoneras... les pusimos el sexypack, que traía lubricante, no sé qué más cosas, un condón, un dedal, no recuerdo que más traía y eran de cajas de cigarros, las pintamos muy bonitas con el signo de mujer-mujer. (Fátima, 40 años, licenciatura, lesbiana, Distrito Federal)

\section{Conclusiones}

Los resultados de esta investigación no pretenden ser generalizados, ni universales, por el contrario, se intenta dar cuenta de la complejidad de factores que interactúan en la construcción de la experiencia sexual de las mujeres, en el cruce con sus interseccionalidades concretas; por lo que además de compartir una condición de género y orientación sexual, las situaciones particulares de las participantes dan cuenta de distintas formas y niveles de opresión, tal como lo refiere Marcela Lagarde (2011). Este análisis y reflexiones son un intento de generar conocimiento que pueda alimentar nuevas rutas de diálogo y acción, que contribuyan al desmantelamiento del sistema heteropatriarcal que regula y nulifica la vivencia plena de las sexualidades en las mujeres.

A través de la experiencia compartida por las entrevistadas es posible dar cuenta de que la educación entorno a la sexualidad está construida dentro de un marco patriarcal y heteronormativo que normaliza y reduce la sexualidad de las mujeres a una lógica reproductiva (al introducir el tema de prevención de embarazos), así como al binomio de salud-enfermedad (al hablar de vih/sida e infecciones de transmisión sexual). En este sentido, los discursos disponibles en torno al cuerpo, el placer, el erotismo y la diversidad sexual, entre otros temas, no forman parte de este espacio, siendo colocados como menciona Rubin (1989) en la 
zona del sexo malo. Es así que se ha invisibilizado la sexualidad e identidad de las mujeres lesbianas y bisexuales dentro de la educación sexual formal.

Con ello se hace evidente la dicotomización de los cuerpos y la obligatoriedad heterosexual, dando como resultado una censura, represión, discriminación y violencia de la existencia de las lesbianas y mujeres bisexuales, y de manera central de su sexualidad.

Ante dicho contexto mencionaron dos estrategias para afrontarlo, en un primer momento la autocensura con la finalidad de continuar con la interacción social que mantenían hasta el momento y, en segundo lugar, la búsqueda de espacios abiertos a la existencia lésbica y bisexual. En el primer caso, se hace evidente los privilegios derivados de la heterosexualidad, mientras que en el segundo, si bien existe una renuncia a dicho reconocimiento por parte del sistema patriarcal y heteronormativo, da lugar a la posibilidad de explorar los deseos e identidades.

Cabe destacar que la mayoría de las mujeres entrevistadas que han obtenido información sobre la sexualidad, identidad y experiencia de las mujeres lesbianas y bisexuales ha sido desde la compartición de saberes en grupos de reflexión y talleres brindados por mujeres que comparten la misma orientación sexual.

Con ello se hace evidente, como ya lo decía Rubin (1989), la sexualidad de las mujeres lesbianas y bisexuales se coloca en el límite de la respetabilidad, con ello es sancionada y censurada, produciendo discursos que obstaculizan a las mujeres vivir el deseo hacia otras mujeres.

Para finalizar, a partir del análisis realizado con las experiencia de estás once mujeres se propone ampliar los contenidos sobre sexualidad en el ámbito educativo en cuatro sentidos, en primer lugar, hacer visible la experiencia sobre la diversidad sexual y de género con la finalidad de ampliar los discursos disponibles y la experimentación; en segundo lugar, ampliar la visión de prevención de infecciones de transmisión sexual y vih/sida a la diversidad sexual y de género para tener una visión incluyente; en tercer lugar, facilitar discursos sobre el placer, erotismo y corporalidad que permitan romper la dicotomía sexualidad buena vs mala; y por último, la sexualidad como un derecho para fomentar la ciudadanía sexual. Para ello se considera necesario que el personal docente sea sensibilizado y capacitado en dichos temas.

\section{Referencias}

AMUCHÁSTEGUI, A. y RIVAS, M. Los procesos de apropiación subjetiva de los derechos sexuales: notas para la discusión. Estudios Demográficos y Urbanos, 543597. 2004. 
CAMPERO, L.; KENDALL, T.; CABALLERO, M.; MENA, A. y HERRERA, C. El ejercicio de los derechos sexuales y reproductivos: un estudio cualitativo de personas heterosexuales con VIH en México. Salud pública de México, 52 (1), 61-69. 2010.

CARÍAS, A. Ejercicio de derechos sexuales y reproductivos en adolescentes. Centro de Derechos de Mujeres-CDM. Tegucigalpa, Honduras: Centro de Derechos de MujeresCDM y Oxfam Internacional. 2006.

CASTAÑEDA, M. El machismo invisible. México: Grijalbo. 2002.

CERVANTES-CARSON, A. y CITERONI, T. Derechos sexuales y la desarticulación del heterosexismo: tolerancia, reconocimiento y liberación. En I. Szasz, \& G. Salas (Coords.), Sexualidad, derechos humanos y ciudadanía: diálogos sobre un proyecto en construcción (p. 303-346). México: El Colegio de México. 2008.

CORRÊA, S. Cruzando la línea roja: cuestiones no resueltas en el debate sobre los derechos sexuales. En I. Szasz, \& G. Salas (Coords.), Sexualidad, derechos humanos y ciudadanía (p. 25-55). México: El Colegio de México. 2008.

FIGUEROA, J. Aproximación al estudio de los derechos reproductivos. México: EI Colegio de México. 1995.

FOUCAULT, M. Historia de la sexualidad I. La voluntad de saber. México: Siglo XXI. 2011.

GIRARD, F. Negando los derechos sexuales y la orientación sexual en la ONU. En R. Parker, R. Petchesky, y R. Sember (Coords.), Políticas sobre sexualidad: Reportes desde las Líneas del Frente (p. 347-398). Lima: Fonte. 2009

GÜIDA, C.; RAMOS, V. Y VITALE, A. Conocimiento y ejercicio de los derechos sexuales y de los derechos reproductivos. En A. López (Coord.), Proyecto género y generaciones: reproducción biológica y social de la población uruguaya. Tomo I estudio cualitativo (p. 247-338). Uruguay: UNFPA. 2006.

KVALE, S. Las entrevistas en investigación cualitativa. Madrid: Morata. 2008.

LAGARDE, M. Los cautiverios de las mujeres: madres, esposas, monjas, putas y locas. México: Universidad Nacional Autónoma de México. 2011.

ORTIZ-ORTEGA, A.; RIVAS-ZIVY, M.; HUERTA, F.; SALAZAR, G. y GÓMEZGONZÁLEZ, A. El papel de los hombres en el ejercicio y apropiación de los derechos sexuales y reproductivos de las mujeres. Perinatol Reprod Hum, 18(1), 2333. 2004.

PLATERO, R. La interseccionalidad como herramienta de estudio de la sexualidad. En R. Platero, Intersecciones: cuerpos y sexualidades en la encrucijada (p. 15-72). Barcelona: Bellaterra. 2012

PLATERO, R. ¿Es el análisis interseccional una metodología feminista y queer? En I. Mendia, M. Luxán, M. Legarreta, G. Guzmán, I. Zirion, y J. Azpiazu. Otras formas de (re)conocer. Reflexiones, herramientas y aplicaciones desde la investigación feminista (p. 79-96). Bilboa: Hegoa, Simref. 2014. 
RODRÍGUEZ G. Treinta años de educación sexual en México. En: M. Mícher (Coord.) Población, desarrollo y salud sexual y reproductiva (p.13-28). México: Grupo Parlamentario del PRD Cámara de Diputados Congreso de la Unión LIX Legislatura. 2004.

RUBIN, G. Reflexionando sobre el sexo: notas para una teoría radical de la sexualidad. En C. Vance, Placer y peligro: explorando la sexualidad femenina. (p. 113190). Madrid: Talasa. 1989.

SÁNCHEZ, A. Cuerpo y sexualidad, un derecho: avatares para su construcción en la diversidad sexual. Sociológica, 24(69), 101-122. 2009.

SÁNCHEZ, J. La creación feminista de los derechos sexuales y reproductivos y la ciudadanía de las mujeres en México. México D.F.: Tesis de Doctorado en Sociología UNAM, Facultad de Ciencias Políticas y Sociales, 2002.

WEEKS, J. Sexualidad. México: Paidós. 1998. 